\title{
High resolution temperature and density profiles during the energy quench of density limit disruptions in Rijnhuizen tokamak project
}

\author{
F. Salzedas, ${ }^{\text {a) }}$ S. Hokin, ${ }^{\text {b) }}$ F. C. Schüller, A. A. M. Oomens, and the RTP Team \\ FOM-Instituut voor Plasmafysica Rijnhuizen, Association Euratom-FOM, Trilateral Euregio Cluster, \\ Nieuwegein, The Netherlands
}

(Received 18 January 2002; accepted 1 May 2002)

\begin{abstract}
Measurements of the electron temperature, $T_{e}$, and density, $n_{e}$, during the energy quench of a major disruption showed that the onset of $T_{e}$ erosion in the neighborhood of the $m / n=2 / 1 \mathrm{O}$ point at the low field side (LFS) accelerates the well-known $m / n=1 / 1$ erosion of the core temperature. During this phase $T_{e}(r)$ is only partially flat in the region between the $q=2$ and the $q=1$ surfaces and $n_{e}(r)$ decreases in the core and increases inside the $m / n=2 / 1$ island. Immediately after the flattening of $T_{e}(r)$ a large peak in $T_{e}$ and to a lesser extent in $n_{e}$ has been observed. This peak is radially localized at the $q=2$ radius at the LFS, is very short lived and is poloidally asymmetric. $T_{e}$ profiles measured by the heterodyne radiometer and the Thomson scattering agree very well up to the time $T_{e}(r)$ flattens but afterwards can be a factor of two different. (C) 2002 American Institute of Physics. [DOI: 10.1063/1.1489675]
\end{abstract}

\section{INTRODUCTION}

The power yield of a tokamak reactor increases with plasma density. However, since the beginning of tokamak research, it has been found ${ }^{1}$ that high density plasmas tend to be unstable and can be abruptly lost during a so-called plasma major disruption. In the small tokamaks available at the time, disruptions only resulted in an early termination of the discharge. As the size of the tokamaks and also their total plasma energy increased it started to be clear that major disruptions are a threat to the structural integrity of a tokamak reactor. In today's tokamak operation a careful choice of the plasma parameters has decreased significantly the occurrence of disruptions and the related damaging effects. However, every disruption is undesirable and the goal is to decrease the probability of occurrence of disruptions as close to zero as possible. So the study of major disruptions is of great importance for the future of tokamak reactors.

Disruptions are thought to occur due to the nonlinear interaction of MHD (magnetohydrodynamic) instabilities. Nonetheless, a detailed physical understanding of their time evolution has still not been fully accomplished. ${ }^{2}$ Experimentally, ${ }^{3-11}$ the difficulties in probing the disruption are mainly connected with its extremely short duration (microseconds), unpredictable onset and 3D structure. Also, except for electron temperature and density, local plasma parameters are difficult to measure, or are not measured at all. Theoretically, ${ }^{12-17}$ the description of the disruption is still not unique. Different models address different parts of the disruption but there is no continuous description of its time

\footnotetext{
a)Present address: Centro de Fusão Nuclear, Association Euratom-IST, Lisbon, Portugal; electronic mail: fsal@cfn.ist.utl.pt

b) Alfvén Laboratory Division of Fusion Plasma Physics, Association Euratom-NFR, Royal Institute of Technology, Stockholm, Sweden.
}

evolution. These difficulties just reflect the complex nature of the phenomenon.

Density limit disruptions are preceded by a sequence of events, called the precursor of the disruption. This sequence is not exactly the same in all tokamaks or even in the same tokamak from disruptive discharge to disruptive discharge. Despite the large difference in sizes and the different geometries in today's tokamaks, density limit disruptions show common features. Typically an $m / n=2 / 1$ tearing mode is destabilized by the contraction of the current profile. At some instant during the mode evolution when its amplitude is large, the energy confinement of the plasma is abruptly destroyed. This process is usually called the energy quench (EQ). So far observations show that it is irreversible, contrary to the course of events that occur during the precursor, which can be avoided. The EQ signals the beginning of the disruption, but it will be shown later that there is not a precise time for its onset, when it is measured with high time resolution. The erosion of the electron temperature profile has been observed in different tokamaks ${ }^{3,5,18,19}$ to have an $m / n=1 / 1$ structure. We will show in this paper that this $m / n=1 / 1$ erosion is precipitated by an off-axis erosion starting from the O point of the $m / n=2 / 1$ mode at the low field side (LFS). The EQ triggers a sequence of events that are also common to all tokamaks, namely the fast increase in poloidal flux with its associated negative loop voltage, $V_{\text {loop }}$, and increase in plasma current, $I_{p}$, and the subsequent resistive decay of $I_{p}$, known as the current quench (CQ) (see Fig. 1).

In this paper we study how the abrupt loss of energy confinement in RTP plasmas, preceded by a $m / n=2 / 1$ mode, takes place. The growth of this mode was described in detail in Refs. 20 and 21. Here the discussion will focus on the time interval between the last moments of the $m=2$ growth and the time at which the plasma current starts to decay, 

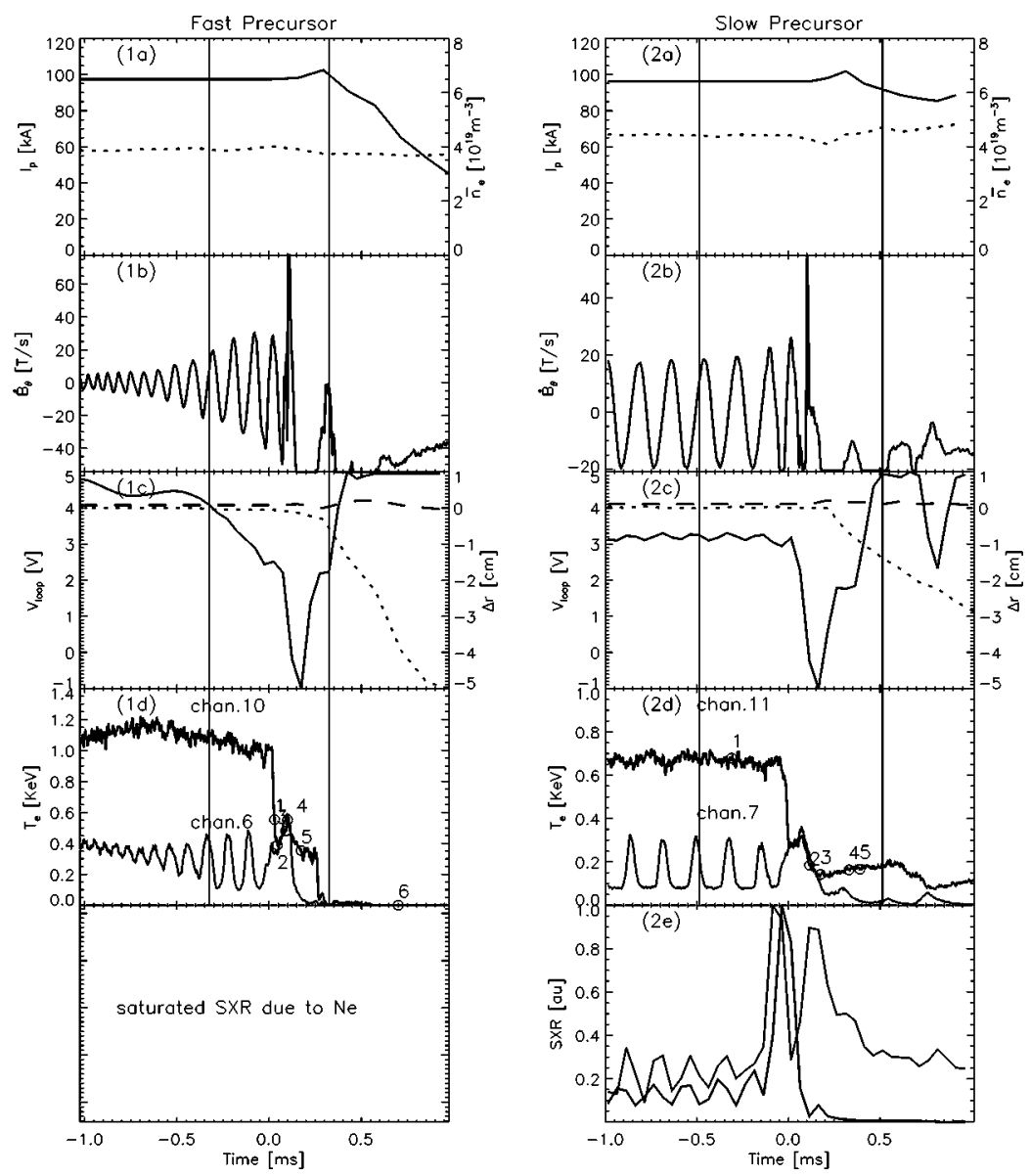

FIG. 1. Illustration, for the cases of FPD and SPD, of (a) plasma current (solid line) and average line integrated density (dotted line). (b) $\dot{B}_{\theta}$ at the equatorial plane at the LFS. (c) Loop voltage (solid line), horizontal (dotted line) and vertical (dashed line) plasma displacement. (d) $T_{e}$ from ECE radiometer. The circles indicate the time of the TS profiles on the respective Figs. 2 and 4. (e) SXR signals. The evolution of $T_{e}(r)$ between the time interval limited by the two vertical grey lines is shown in Figs. 2 and 4 for the cases of FPD and SPD, respectively. All signals are synchronized in time.

indicated in Fig. 1 by two vertical gray lines (Secs. III and IV). In Sec. V we compare the EQ in minor disruptions and major disruptions. Next, a short description of the experimental setup and of the plasmas here studied will be given.

\section{EXPERIMENTAL SET-UP}

The innocuous effects of disruptions in small tokamaks make these very suitable for their study. Besides its small size, RTP (Rijnhuizen tokamak project, minor radius $a$ $=0.16 \mathrm{~m}$, major radius $R_{0}=0.74 \mathrm{~m}, B_{\phi}<2.5 \mathrm{~T}$. and $I_{p}$ $<150 \mathrm{kA}$ ) was equipped with a set of very good diagnostics that allowed us to probe the energy quench with high spatial and temporal resolution.

The time evolution of $T_{e}(r)$ with $1 \mu$ s time resolution, along a horizontal chord throughout the equatorial plane, is measured with a 20 channel electron cyclotron emission (ECE) radiometer. Perpendicularly, along a vertical chord, $T_{e}(r), n_{e}(r)$, and $p_{e}(r)$ profiles with $3 \mathrm{~mm}$ spatial resolution were measured by Thomson scattering (TS). In order to scan the energy quench with TS, this diagnostic was triggered by a signal proportional to the amplitude of $\widetilde{B}_{\theta, m=2}$.

In RTP there are two types of precursors. ${ }^{20}$ The first type is mainly characterized by an exponential increase of $\widetilde{B}_{\theta, m}=2$ for a short period of $\approx 0.5 \mathrm{~ms}$ prior to disruption. ${ }^{21}$ In the second type, $\widetilde{B}_{\theta, m=2}$ stays quasisaturated for a period that can go up to $100 \mathrm{~ms}$ prior to disruption. Disruptions with these two precursors will be analyzed in parallel, in order to show their similarities and differences. Henceforth we will refer to these disruptions simply as fast precursor disruptions (FPD) and slow precursor disruptions (SPD), respectively. In both cases $q_{a} \simeq 4$ with $95 \mathrm{kA} \leqslant I_{p} \leqslant 100 \mathrm{kA}$ and $2.08 \mathrm{~T} \leqslant B_{\phi}$ $\leqslant 2.16$ T. For those with fast precursors, the density was increased by puffing $\mathrm{Ne}$ gas in a He plasma and for the slow precursors He gas was puffed in a He plasma. The resulting disruptive events were found to be reproducible to the extent that TS profiles measured during disruptions of separate discharges could be put together to reconstruct a single quench evolution.

Mode locking was never observed, i.e., the mode rotation never came to rest relative to the wall. FPD occur when the $m=2$ mode has a frequency of $\approx 10 \mathrm{kHz}$. In SPD, the frequency is lower, between $5-6 \mathrm{kHz}$.

In Figs. 2(a) and 4(a) the evolution of $T_{e}(r)$ measured by the ECE radiometer is shown for the cases of FPD and SPD, respectively. With the purpose of simplifing the discussion, in the two pictures the time origin $(t=0)$ was set to coincide with the last time the $m=2$, X point passed in front of the ECE radiometer, before the observed fast collapse in $T_{e}$ at the plasma core. The temperature of the isotherms is indicated in $\mathrm{eV}$, and to enhance the contrast two gray areas are used. The dark one is in the vicinity of the central $m$ $=1$ mode boundary, while the lighter one is in the vicinity of the $m=2$ separatrix facing the core. In these figures the position of the top-bottom carbon limiter (at $\rho=1$ ) is marked by the dotted line, to guide the eye. Figure 3 shows a collection of TS profiles measured during an FPD and Fig. 5 is the 


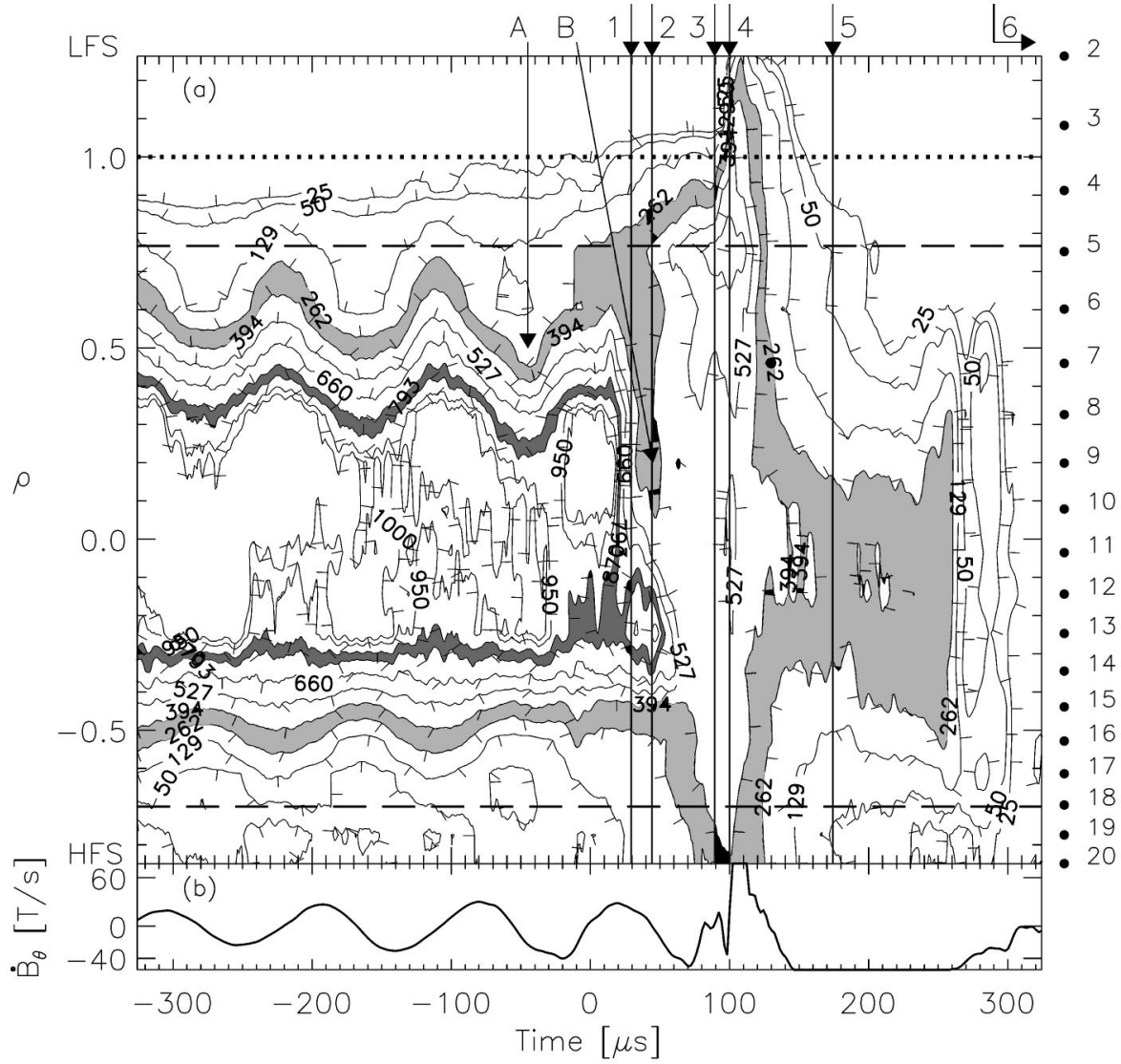

FIG. 2. Illustration of a fast precursor major disruption. (a) Time evolution, with $1 \mu$ s time resolution, of the radial temperature profile measured by the ECE radiometer for the discharge shown in Fig. 1(1), in the time window between the vertical grey lines. The position of the channels is indicated at the right. The arrows indicate the time position of the TS profiles of Fig. 3. TS profile 6 is at $t \approx 700 \mu$ s (see the Appendix). The horizontal dashed lines indicate the position of the $q=2$ surface. The dotted line indicates the position of the limiter. (b) $\dot{B}_{\theta}$ at the equatorial plane at the LFS and displaced a toroidal angle of $150^{\circ}$ from the ECE radiometer.

same for a SPD. The time sequence of the TS profiles is indicated by arrows, in Figs. 2 and 4. As said, the TS profiles belong to different discharges (except for those cases that are indicated). Their position in respect to the quench evolution was chosen with the help of ECE isotherms contour plots. For justification of the choice, the ECE contour plots of the individual discharges are shown in the Appendix together with the real time points of the TS measurements.

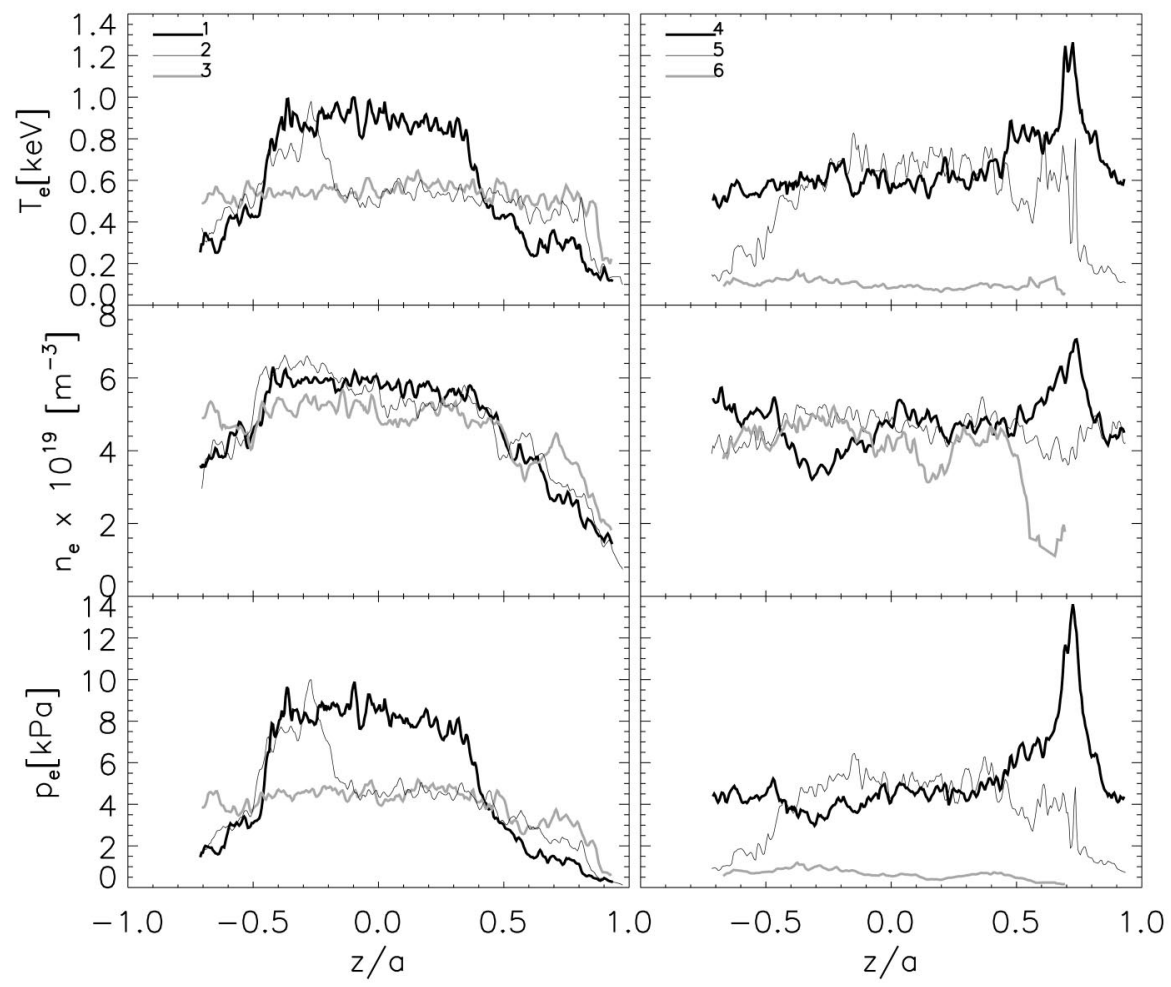

FIG. 3. High spatial resolution $(3 \mathrm{~mm})$, Thomson scattering profiles obtained during the energy quench of fast precursor disruptions. Profiles 1 and 3 , belong to the same discharge. The relative time positions are schematically indicated in Figs. 1(d) and 2(a). The real time positions are shown in the Appendix. 


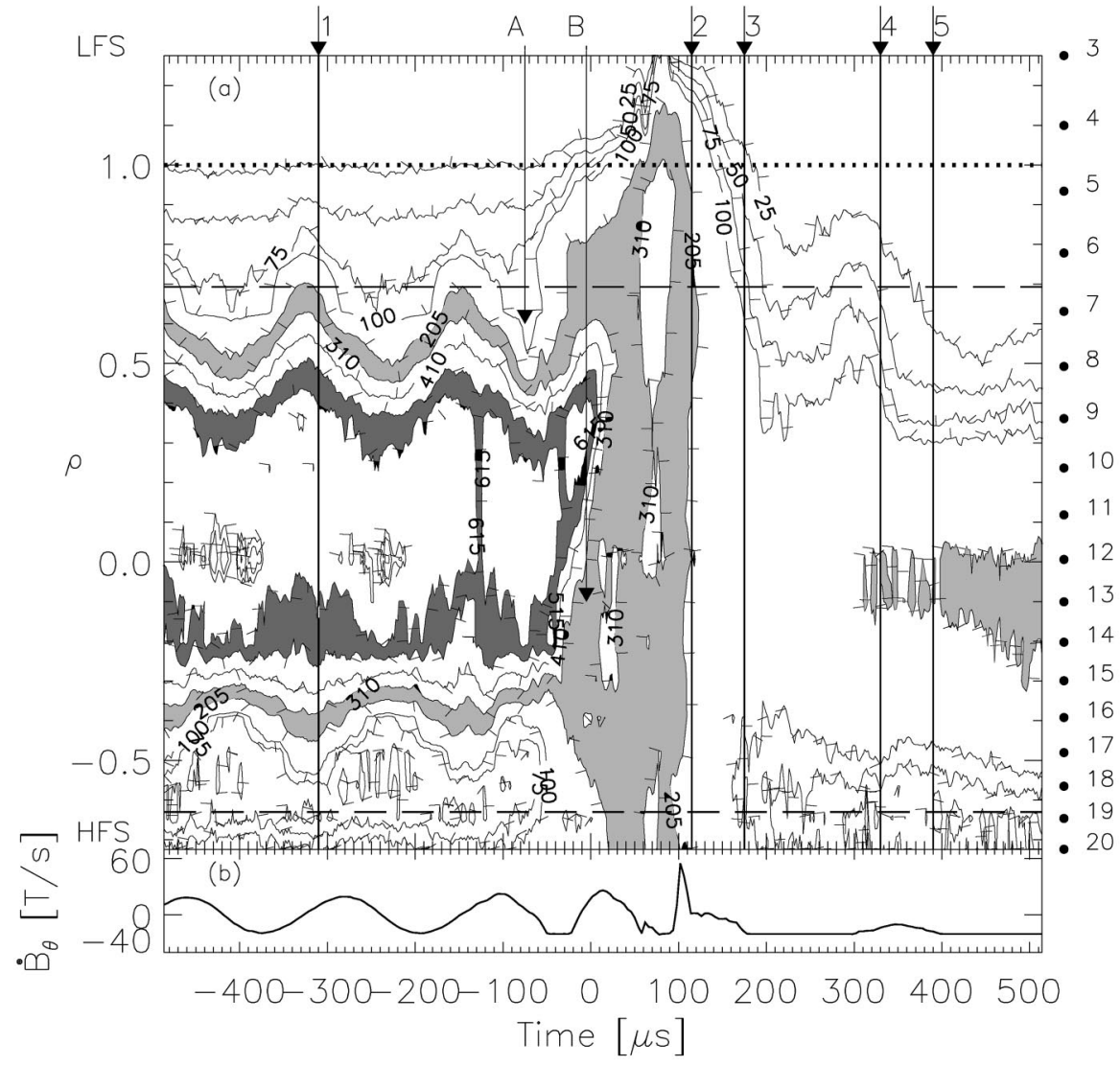

FIG. 4. Illustration of a slow precursor major disruption. (a) Time evolution, with $2 \mu$ s time resolution, of the radial temperature profile measured by the ECE radiometer for the discharge shown in Fig. 1(2), in the time window between the vertical grey lines. The position of the channels is indicated at the right. The arrows $1-5$ indicate the time position of the TS profiles of Fig. 5. The horizontal dashed lines indicate the position of the $q=2$ surface. The dotted line indicates the position of the limiter. (b) $\dot{B}_{\theta}$ at the equatorial plane at the LFS and displaced a toroidal angle of $150^{\circ}$ from the ECE radiometer.

\section{THE FIRST PHASE OF THE ENERGY QUENCH UNTIL THE FLATTENING OF $T_{e}(r)$}

Erosion of the central temperature profile is first visible around $t=-250 \mu \mathrm{s}$ at the cold point of the central $m=1$ mode at the high field side (HFS) [see Fig. 2(a)]. The heat lost in the core flows to the edge leading to the expansion of the temperature profile first visible at the LFS and somewhat later at the HFS. The same $m / n=1 / 1$ like erosion has been

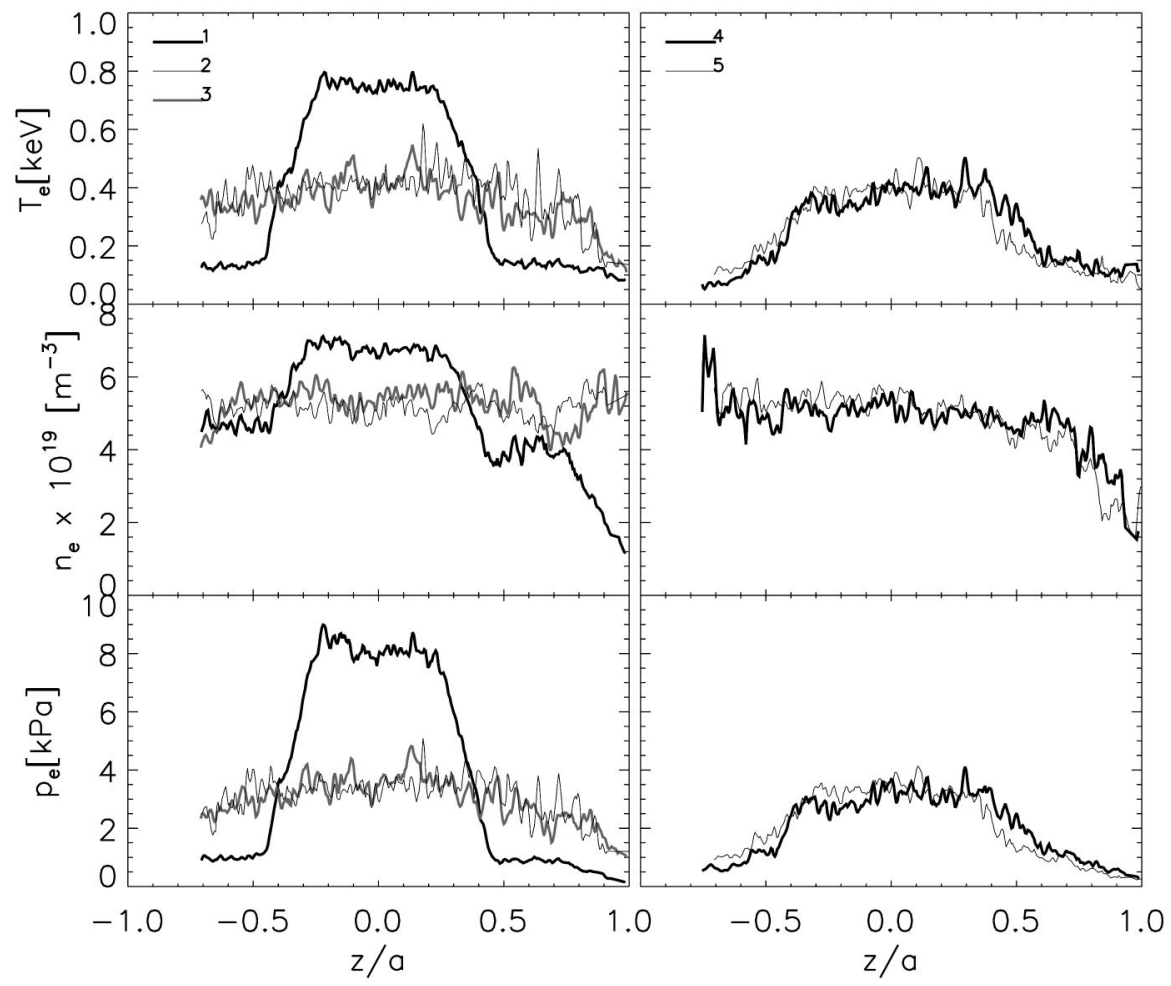

FIG. 5. High spatial resolution ( $3 \mathrm{~mm}$ ) Thomson scattering profiles obtained during the energy quench of slow precursor disruptions. Profiles 2 and 3, belong to the same discharge, just like profiles 4 and 5 . The relative time positions are schematically indicated in Figs. 1(2d) and 4(a). The real time positions are shown in the Appendix. 
observed in several tokamaks and leads to a crescent shaped remnant of the original hot core with circular cross section. The crescent becomes thinner and thinner during the erosion process. At $t=-50 \mu \mathrm{s}, \rho=0.5$ another erosion process becomes visible which starts to affect the $m=2$ mode by a growing indentation of the core facing separatrix. This indentation describes a slight, excursion towards the plasma core at the angular position of the $m=2$, O point (see arrow A) and it is symmetric in time around the $\mathrm{O}$ point. At $t$ $=0 \mu \mathrm{s}$ in the plasma core, the degradation of energy confinement intensifies, as can be seen by the widening of the dark gray region at $\rho \approx-0.25$. At the same time, the light gray isothermal area expands to inside and outside and crosses the $m=2 \mathrm{X}$ point, advancing towards the plasma edge at the LFS.

From $0-40 \mu \mathrm{s}$, as the $m=2$ mode passes in front of the ECE radiometer, it is observed that erosion of the $T_{e}$ profile is already significant (see arrow B). The temperature at the cold point of the $m=1$ mode decreased $50 \%$ and has become equal to the temperature at the $m=2, \mathrm{O}$ point, at the LFS. As seen in the first TS profile in Fig. 3, $\left(T_{e 1}^{\mathrm{TS}}\right)$ the temperature profile inside the hot $m=1$ island as well as inside the $m$ $=2$ island is irregular. In $T_{e 2}^{\mathrm{TS}}$ the profile is flattened between the $m=2$, X point and the $m=1$, hot point, only for positive values of $z / a$. After this, within $40 \mu$ s the temperature of the $m=1$ hot point decreased $50 \%$ and the $T_{e}$ profile becomes completely flat, up to $\rho=0.9$ (see $T_{e 3}^{\mathrm{TS}}$ ).

From these observations it is seen that it is not possible to ascribe a precise starting time for the onset of the energy quench. The degradation of the energy confinement is a continuous process that becomes more intense during the period between -50 and $+90 \mu \mathrm{s}$, as shown by the excursion of the isotherms at the edge. This phase of faster erosion of $T_{e}(r)$ is observed to start from the LFS in between the $m=2$, O point and the $m=1$ cold point (arrow A) proceeding asymmetrically into the plasma core, first through the $m=1$ cold point and then to the $m=1$ hot point (arrow B).

Despite the different evolution of $\widetilde{B}_{\theta}$, a similar sequence of events occurs for the case of the SPD, Fig. 4. One apparent difference between SPD and FPD is due to the mode rotation frequency that is almost two times smaller for SPD. In this way, since the erosion of the $m=1$ cold point occurs in approximately the same time as in the FPD case $(\approx 90 \mu \mathrm{s})$, the fact that the mode rotates more slowly results in an erosion that occurs within half a toroidal rotation of the $m=2$ mode. So the beginning of the $m=2 \mathrm{O}$ point erosion (arrow A) is not always observed, since it depends on which part of the $m=2$ mode is passing in front of the ECE radiometer in this period (see, for example, Fig. 11 in the Appendix).

From Thomson scattering, the behavior of the electron density profile could be followed. For the FPD cases it is observed in Fig. 3 that from $n_{e 1}$ to $n_{e 3}$ the central density decreases $\approx 20 \%$ in the plasma core and increases $\approx 50 \%$ inside the $m=2$ island. Observing in sequence the first three $n_{e}$ profiles, in the region between $z / a \approx 0.5$ to $z / a \approx 0.8$, it is apparent that there is a perturbation in the density gradient moving outwards. This indicates that the density increase in the $m=2$ island could be the result of the flow of particles from the island neighborhood in the core side. We should note that such an outward displacement of density perturbations is already apparent in the last stages of the fast precursor $^{21}$ as is the density increase inside the $m=2$ islands.

In DIII-D $n_{e}$ was observed to have a similar behavior, ${ }^{10}$ where an increase in $n_{e}$ at $r / a \approx 0.75$ is observed in a TS $n_{e}$ profile taken during the major EQ (see Fig. 2 of Ref. 10 at $2.0444 \mathrm{~s}$ ). The $m$ number, as well as the radius of the resonant surface of the $n=1$ locked mode that precedes the disruption, is not mentioned so we cannot conclude that it is related to the density increase inside the $m=2$ island observed at RTP. However, in DIII-D it was also observed that an $n_{e}$ decrease in the core is followed by an $n_{e}$ increase in the plasma edge.

In the center of the profiles $n_{e 2}$ and $n_{e 3}$ a step in the density is visible. For $n_{e 2}$ the highest density is between $z / a \approx-0.5$ to $z / a \approx 0$, which corresponds to the hot part of the $m=1$ mode. The cause of this step is not understood. For $z / a>0.8$ it is observed that the density does not change significantly. So, during this period of the energy quench despite this internal rearrangement of the electron density, the global convex shape of $n_{e}(r)$ is still more or less preserved, as was previously observed. ${ }^{22}$

The soft $\mathrm{x}$-ray (SXR) diagnostic was not tuned for this type of discharge and often several channels were saturated. In the case of the discharge shown in Fig. 4, the intensity of the SXR radiation a few milliseconds before the disruption was low enough to allow the measurements shown in Fig. $1(2 \mathrm{e})$. The viewing chords of the two channels intercept at $r / a=0.65, z / a=0.75$, that is, approximately the radius of the $q=2$ surface in the LFS. The first spike in the SXR, visible on both channels, occurs when the $m=2, \mathrm{X}$ point is at the LFS. Its origin is probably related with the increase at the $m=2$, X point temperature that occurs at $t=0$ as described before. The second spike is only visible at one channel which means that the enhanced emissivity at the $m=2 \mathrm{X}$ point dies out very quickly and asymmetrically. The same behavior of SXR is observed for minor disruptions, and that will be discussed in Sec. V.

\section{THE SECOND PHASE OF THE ENERGY QUENCH AFTER THE FLATTENING OF $T_{e}(r)$}

Shortly after the flattening of $T_{e}(r)$, i.e., when the $m$ $=1$ erosion is completed, a series of new phenomena is observed.

The remaining gradients in $T_{e}(r)$ and $n_{e}(r)$ at the plasma edge disappear.

The appearance of a short-lived intense peak in the temperature and to a lesser extent in the density at a radius between $0.7<\rho<0.8$.

An increasing deviation between $T_{e}$ measured by TS and ECE can be noticed while normally these two diagnostics are very well in agreement with each other. 


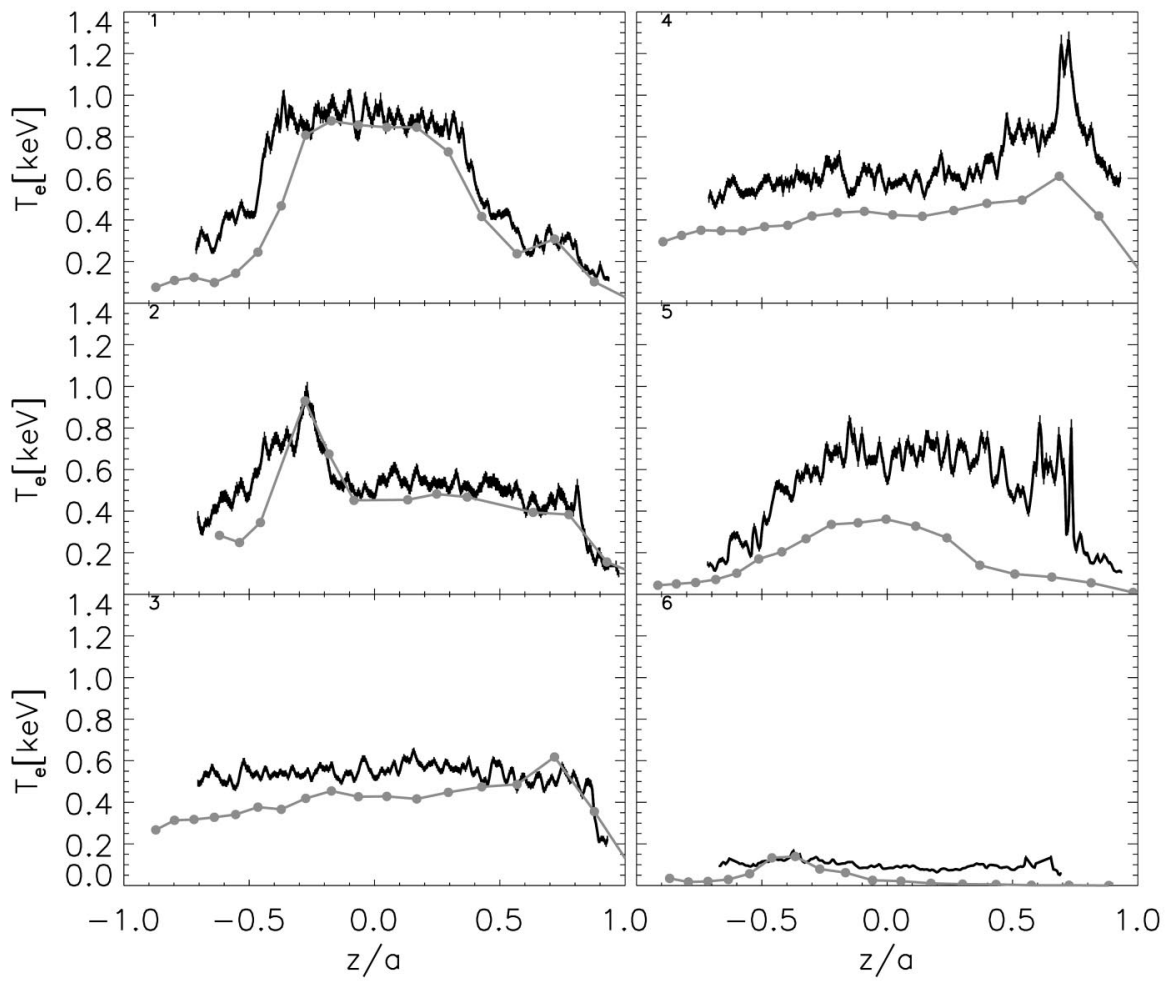

FIG. 6. Comparison between the $T_{e}^{\mathrm{TS}}$ profiles of Fig. 3 and the $T_{e}^{\mathrm{ECE}}$ profiles measured at the same time. It is recalled that the TS profiles are measured along a vertical chord, perpendicular to the measuring chord of the ECE radiometer. Until the flattening of $T_{e}$ the two diagnostics agree very well. After the flattening of $T_{e}$ the electron temperature measured by Thomson scattering is always larger. For the profiles 5 the difference in the central temperature is already $50 \%$.

\section{A. Disappearance of gradients}

The disappearance of the $T_{e}$ gradients at the edge is only visible in $T_{e}^{\mathrm{ECE}}$. In Fig. 2(a) (FPD) it occurs between $t=+70 \mu \mathrm{s}$ and $t=+100 \mu \mathrm{s}$. The TS profile 4 does not cover the very bottom edge of the plasma and at the top the peak masks the effect. In Fig. 4(a) (SPD) the edge gradients disappear between +20 and $+80 \mu \mathrm{s}$.

After the flattening of $T_{e}$, the electron density remains
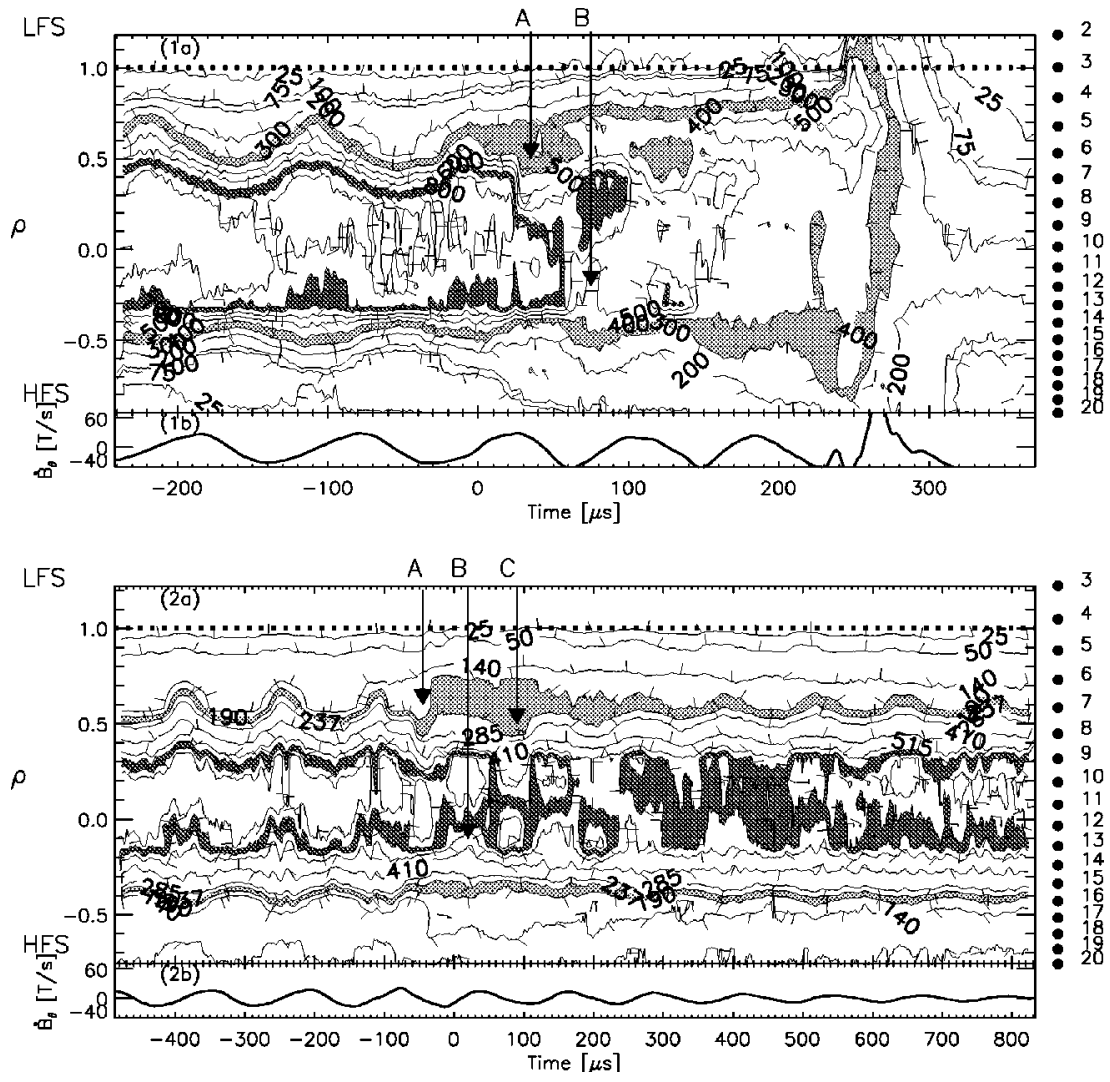

FIG. 7. (1a)-(1b) Example of a fast precursor disruption where the erosion of the core temperature lasts for a period more than two times longer than the normal cases exemplified in Figs. 2 and 4. (2a)-(2b) Illustration of a minor disruption that occurred before a SPD. 
high (see Figs. 3 and 5). However, the $n_{e}$ profile is no longer convex but flat or even slightly hollow. The gradients between $\rho=0.5$ and 1 have disappeared. Except for the peak in $n_{e 4}$ in Fig. 3, the other profiles show large perturbations. Their occurrence is erratic with no apparent correlation with other observations. This fact together with the large size of these perturbations suggests that large scale turbulence inducing large scale convection in the plasma may be taking place during this last phase of the energy quench.

\section{B. The peak in Te}

Atapproximately $100 \mu \mathrm{s}$ an increase in $T_{e}^{\mathrm{ECE}}$ at $\rho \simeq 0.77$ is observed in Fig. 2(a). This peak in $T_{e}^{\mathrm{ECE}}$ is localized at the same radius as the $m=2$ island. As shown in Fig. 6(4) the peak is observed simultaneously at the top by TS and at the LFS by the ECE radiometer. It was also observed simultaneously at the LFS by the ECE and at the bottom by TS. However, as is shown in the Appendix, this peak was never observed by ECE at the HFS. This means that poloidally it spans an angle that is between $90^{\circ}$ and $180^{\circ}$ at the LFS. The peak value of the temperature measured in $T_{e 4}^{\mathrm{TS}}$ is two times higher than the average electron temperature in the plasma center at the same point in time and it is $20 \%$ higher than the central temperature before the disruption [see Fig. 9(2) in the Appendix]. The ECE radiometer measures a peak temperature of only $600 \mathrm{eV}$ when TS measures $1200 \mathrm{eV}$. It should be stressed that this peak is observed in all disruptions, as shown in the Appendix, so it must be an intrinsic phenomenon related to disruptions.

The electron density, that was already increasing in the previous profile $n_{e 3}^{\text {TS }}$ in the $m=2$ island, also peaks in the same position as the temperature, although less pronounced. Another characteristic of the peak is found in the Thomson scattering spectrum which at that place shows a pronounced high energy tail that is not visible at other positions of the viewing chord. This indicates the presence of fast electrons.

Relative to the magnetic poloidal field, the $m=2$ oscillation is destroyed at the time the peak is observed. This is a strong indication that the $m=2$ island is also destroyed. But, it is not clear if it is the destruction of the island that provokes the peak or if it is the peak that provokes the destruction of the island. It should be noted that the poloidal asymmetry of the peak indicates that it has no $m / n=1 / 1$ topology.

\section{Difference between TS and ECE}

Under all normal circumstances in RTP, even during electron cyclotron resonance heating, the ECE temperatures after correction for optical depth were in excellent agreement with values derived from TS. As shown in Fig. 6, after the flattening of $T_{e}(r)$ a major discrepancy between the ECE radiometer and the TS develops, becoming as large as a factor of 2, with the ECE radiometer measuring always the lowest values. The measured values of the electron density are much lower than the cutoff value for the ECE. So this effect cannot cause the observed discrepancy. The cause may be in deviations from Maxwellian distribution, since the discrepancy starts only after the flattening of $T_{e}$, during the onset of the large variations in the loop voltage.
The negative spike in the loop voltage occurs between 50 and $150 \mu \mathrm{s}$ in both examples given here [see Figs. 1(c) and $2(\mathrm{c})]$.

In the same period the oscillations of $d B / d t$ are interrupted with a large spike, as shown in Figs. 2(b) and 4(b) and the outer isothermal surfaces show the largest expansion, as can be seen at $\approx 100 \mu \mathrm{s}$.

\section{MINOR DISRUPTION AND SLOWER MAJOR DISRUPTION}

The continuous degradation of energy confinement that occurs during the energy quench, up to the flattening of the $T_{e}$ profile, as described in Sec. III, can be interrupted. When this happens the energy confinement improves again allowing the electron temperature to raise, returning to previous values. An example of this process, called a minor disruption, is shown in Fig. 7(2). The mechanism that during a minor disruption prevents the plasma to evolve to a major disruption is not known. In this section we address this problem heuristically, underlining the behavior of the electron temperature in the region of the $q=2$ surface.

To better illustrate our point, we show in Fig. 7(1) a major disruption where the core $T_{e}$ erosion lasted for a period more than two times longer than the two (typical) examples shown before. In this example it is observed that the equivalent process of erosion described before at the position of arrow A, Fig. 2(a), is preceded by a seemingly larger outward heat flow through the $m=2 \mathrm{X}$ point, than previous examples, as is indicated by the excursion of hot isotherms $[200 \mathrm{eV}$ and $300 \mathrm{eV}$ in this example in Fig. 7(1)] through the $m=2$, X point, from the core facing side ${ }^{30}$ of the island to the edge facing side, at the LFS.

This causes the $m=2 \mathrm{O}$ point erosion, occurring at the position of arrow $\mathrm{A}$, to start at higher temperature and to evolve more slowly to the plasma core. The $m / n=1 / 1$ erosion proceeds in a similar way as the cases described before [note arrow B in Fig. 7(1)], but the thermal energy is kept longer in the region inside the $q=2$ radius (the light gray isothermal region only reaches the core at $t=220 \mu \mathrm{s}$ ).

In the case of the minor disruption, Fig. 7(2), the $m=2$ $\mathrm{O}$ point erosion (arrow A) is preceded also by a larger outward heat flow through the $m=2, \mathrm{X}$ point, like in Fig. 7(1). Such heat flow is not noticeable in the normal major disruptions [compare Fig. 2(a) and Fig. 4(a)]. Between $t=0$ (arrow B) and $t=150 \mu \mathrm{s}$ the $m / n=1 / 1$ erosion increases, but at the $m=2$ mode it is seen that the light gray isothermal region does not extend further inwards (arrow C) or outwards. Afterwards this region shrinks again and in the core the temperature at the $m=1$ mode increases.

In disruptions, the $m=2$ mode behaves like a dynamic heat switch, allowing excursions of the isotherms to the edge, via the $\mathrm{X}$ point, and into the core, via the $\mathrm{O}$ point. In a minor disruption, contrary to the major disruption, the isotherms excursion to the edge is interrupted and heat flows mainly from the $\mathrm{X}$ point into the $\mathrm{O}$ point. This has the effect of shrinking the island and at the same time decreasing the effect that the $m=2$, O point erosion has on the core of the plasma. As the island shrinks, degradation of the energy con- 
finement is stopped and reversed, allowing the $T_{e}$ profile to recover by Ohmic heating. So, from these experiments it appears that the $m=2$ mode plays the critical role in increasing or reducing the rate at which energy confinement in the core is degraded, as changes in the rest of the plasma always occur after the changes in the $m=2$ island. Besides an apparent temperature dependence, it is not possible with the available data to investigate further the structure of this heat switch. Nonetheless three different behaviors can be distinguished.

The switch opens and closes (minor disruption).

The switch opens completely (major disruption).

An intermediate behavior between the previous ones, where the heat switch does not open completely (major disruption with longer quench time).

\section{DISCUSSION AND CONCLUSIONS}

The global scaling of the quench duration with the Lundquist number ${ }^{23}$ indicates that, in the process of nonlinear interaction between modes, the balance dominates between the driving force, being the tension in the magnetic field lines, and the braking force, being magnetic reconnection. However, this balance does not explain the detailed mechanism and cannot predict in fine detail the various differences in quench behavior. A widely spread model for the beginning of the energy quench evokes the stochastization of magnetic field lines between the $m=2$ and the $m=1$ modes. The nonlinear interaction of these two modes would lead to the destabilization of other modes in between, mainly the $\mathrm{m} / \mathrm{n}$ $=3 / 2$ mode, and when the width of the islands equals the distance between them the magnetic field lines would become stochastic destroying the magnetic flux surfaces. The strong point for this model is that it predicts high electron heat diffusivity throughout the stochastic region, which is comparable to the heat fluxes observed during a major disruption. Experimentally, it is not possible to measure stochasticity directly and its presence has to be inferred indirectly. One expected effect is the flattening of the temperature in the regions that have stochastic field lines. A limitation of this model is the lack of a description for the time evolution of the process of stochastization and the lack of a self-consistent computation of the magnetic field and current.

In the measurements previously described, the clearest observation of a flattened temperature profile between the $m=2$ and the $m=1$ mode is in $T_{e 2}^{\mathrm{TS}}$, Fig. 3, where it is observed that between the $m=2, \mathrm{X}$ point at the top and the $m=1$ hot point at the bottom, the $T_{e}$ profile is flat. A closer look into the same profile shows that, although at the top the $T_{e}$ profile is flat up to $z / a=0.8$, at the bottom there is a gradient from $z / a=-0.45$ to $z / a=-0.7$, which is the position of the $q=2$ resonant surface. This means that the neighborhood of the $m=2$ island core facing side is not all at the same temperature. This seems to be at variance with the complete stochastization of this region at this advanced phase of the profile erosion, as expected by some authors. ${ }^{12}$

The corresponding pressure profile, $p_{e 2}$, is more symmetric. It shows a small gradient at $z / a=0.45$, which is due to a corresponding gradient in the density, and at the position of the $q=2$ surface the pressure is equal to $2 \mathrm{kPa}$, both on the top and the bottom. This means that the helical symmetry in the pressure distribution inside the island is much better preserved than in temperature and density, which makes sense from the parallel momentum balance point of view.

There are features in the measurements shown in this paper that can be interpreted as indirect indications of the existence of electron flows. One of these indications comes from the evolution of the density profile during the $T_{e}$ erosion, which shows a decrease in the core and an increase at the $m=2 \mathrm{O}$ point, with the density perturbation moving outwards, opposite to the density gradient. The sequential outward displacement of the $n_{e}$ perturbations intuitively indicates the existence of a flow. However, the cause and 3D structure of such a particle flow is not clear from these measurements. The other indirect indication of the existence of flows comes from the evolution of the $T_{e}$ profiles. In Figs. 2(a) and 4(a), the deformation of the isotherms indicated by the arrow A seems to indicate the presence of an inward (i.e., towards the core) convective motion at the $\mathrm{O}$ point of the $m=2$ mode. Such a motion does not have to be necessarily connected with the others described before. Linear ${ }^{24}$ and nonlinear ${ }^{25}$ MHD theory of tearing modes predicts vortex flows that advect fluid into the $\mathrm{X}$ point and out from the $\mathrm{O}$ point. Although intrinsic to the tearing mode, these are one fluid flows. It has to be investigated if in the conditions of tokamak disruptions, analogous vortex flows of electrons can have the behavior described above. We note, for example, that advances in tearing mode theory ${ }^{26,27}$ predict the decoupling between the electron and ion fluids during reconnection, which allow the electrons to play the important role in the fast transport of energy. Such theory recently obtained support from experiment. ${ }^{28}$ Recent simulations of high $\beta$ disruptions have also found vortex flows involved in the transport of energy during the energy quench. ${ }^{29}$

A very short-lived peak in $T_{e}$ is observed at every disruption. The nature of this peak is not understood. Its radial position, as well as its correlation with $\dot{B}_{\theta}$, relates it to the $m=2$ mode. However, the reason for the helical asymmetry exhibited by the peak is not clear. While in the preceding first part of the quench, at least the helical symmetry of the pressure inside the $m=2$ island seems to be preserved, during the peak even this is lost and it becomes doubtful if an island topology still exists. From the Thomson spectrum, the presence of fast electrons at the time of the peak is inferred. The discrepancy between Thomson and the ECE radiometer temperature profiles that is observed starting at the flattening of $T_{e}$ is also probably due to the presence of the fast electrons. Possibly the destruction of the $m=2$ mode that occurs at the same time that the peak is observed results in the scattering of the fast electrons throughout the plasma volume, inducing in this way the sudden decrease in $T_{e}$ as measured by the ECE radiometer.

After the flattening of $T_{e}$ the kinetic energy is lost at a comparable rate as before the flattening, during the $m=1$ erosion. In this phase, except for the peak, mode structures 
are no longer observed, while the electron density remains high.

From all these observations we note that the $m / n=2 / 1$, $\mathrm{O}$ point $T_{e}$ erosion associated with the density increase inside the $m / n=2 / 1$ island indicates that convection may be involved in the onset of the energy quench.

\section{ACKNOWLEDGMENTS}

F.S. was supported by the Programa PRAXIS XXI-grant BD/4531/94. This work was performed under the EuratomFOM association agreement, with financial support from NWO and Euratom.

\section{APPENDIX: ORIGINAL DISCHARGES OF THOMSON SCATTERING PROFILES \\ Fast precursor disruptions}

The time positions of Thomson scattering profiles from Figs. 2 and 3 are indicated here in the original discharges. (See Figs. 8-10.)
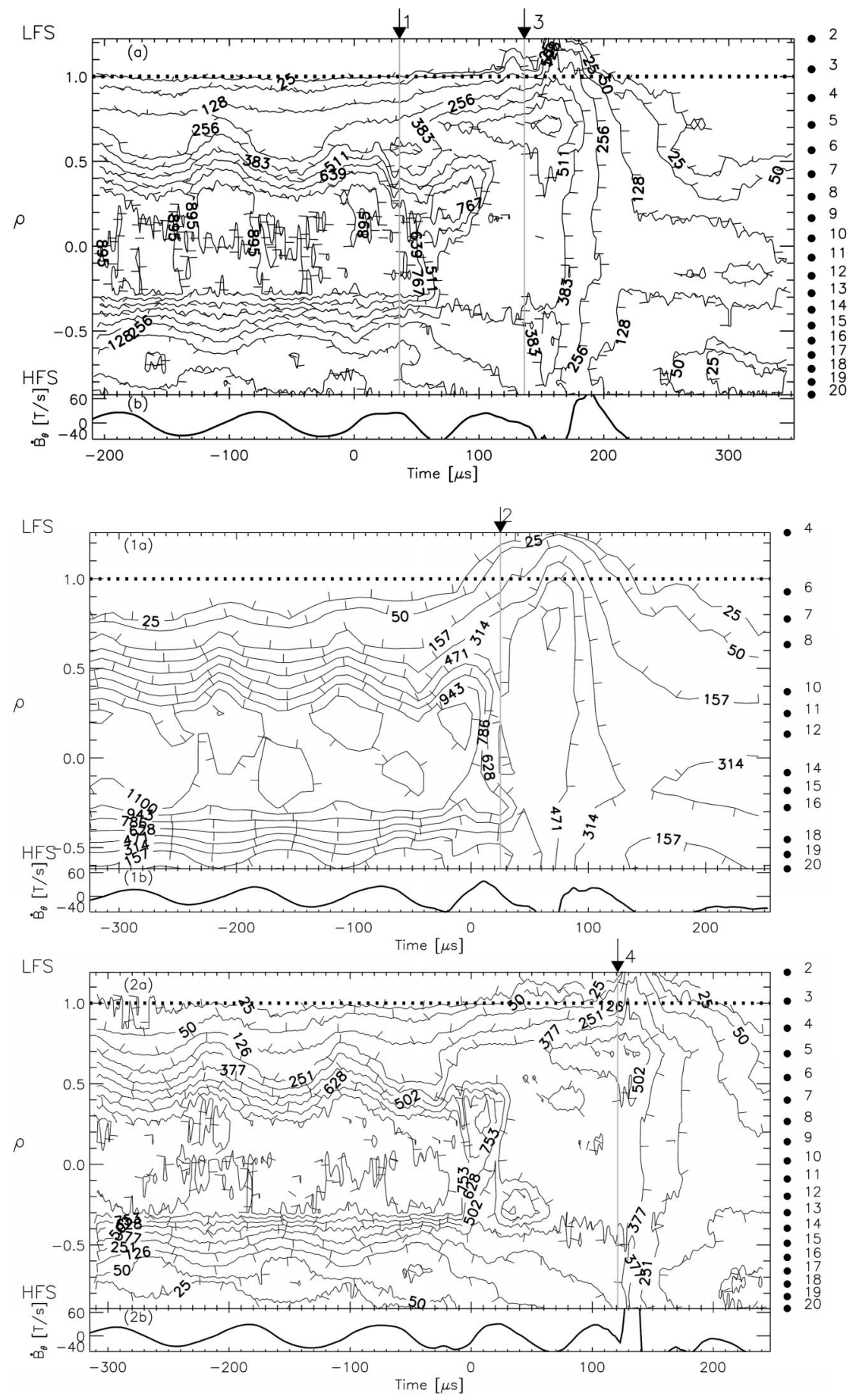

FIG. 8. (a) $T_{e}(\rho, t)$ as measured from the ECE radiometer with $1 \mu$ s time resolution. The position of the channels is shown on the right-hand side. (b) $\dot{B}_{\theta}(t)$ as measured from the fast coil with $2 \mu$ s time resolution. Arrows indicate the time position of TS profiles 1 and 3 from Fig. 3.

FIG. 9. The same as in Fig. 8, except that the ECE time resolution is $10 \mu \mathrm{s}$ in Fig. 1(a). Also in this experiment some channels of the ECE radiometer were not available, as shown on the right-hand side of Fig. 1(a). Arrows indicate the time position of TS profiles 2 and 4 from Fig. 3. 


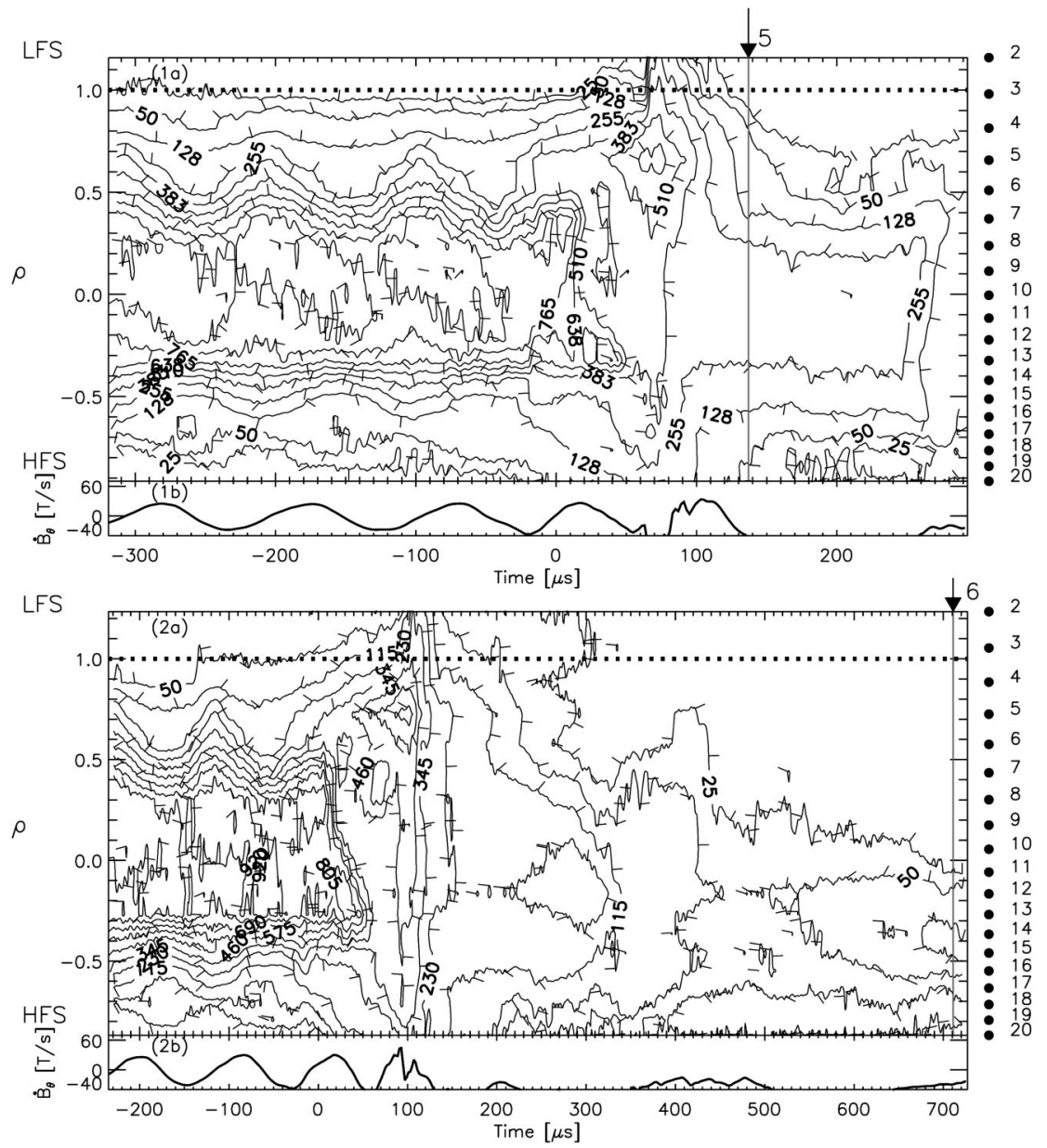

FIG. 10. The same as in Fig. 8. Arrows indicate the time position of TS profiles 5 and 6 from Fig. 3.

\section{Slow precursor disruptions}

The time positions of Thomson scattering profiles from Figs. 4 and 5 are indicated here in the original discharges. (See Figs. 11 and 12.)

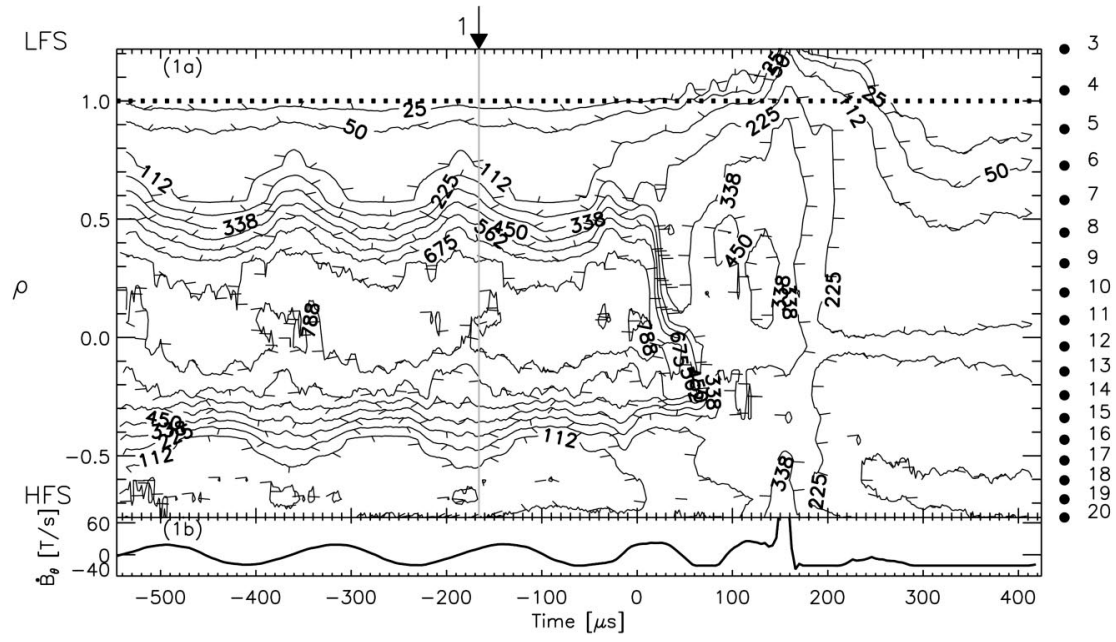

FIG. 11. (a) $T_{e}(\rho, t)$ as measured from the ECE radiometer with $2 \mu \mathrm{s} \mathrm{time} \mathrm{resolution.} \mathrm{The} \mathrm{position} \mathrm{of} \mathrm{the} \mathrm{channels} \mathrm{is} \mathrm{shown} \mathrm{on} \mathrm{the} \mathrm{right-hand} \mathrm{side.} \mathrm{The} \mathrm{initial}$ phase of the evolution of the $T_{e}$ erosion is not visible in this picture. At $t=50 \mu \mathrm{s}$ when the $m=2 \mathrm{O}$ point passes in front of the ECE radiometer, the $T_{e}$ profile of the $m=1$ cold point is already eroded. (b) $\dot{B}_{\theta}(t)$ as measured from the fast coil with $4 \mu$ s time resolution. Arrow indicates the time position of TS profile 1 from Fig. 5. 


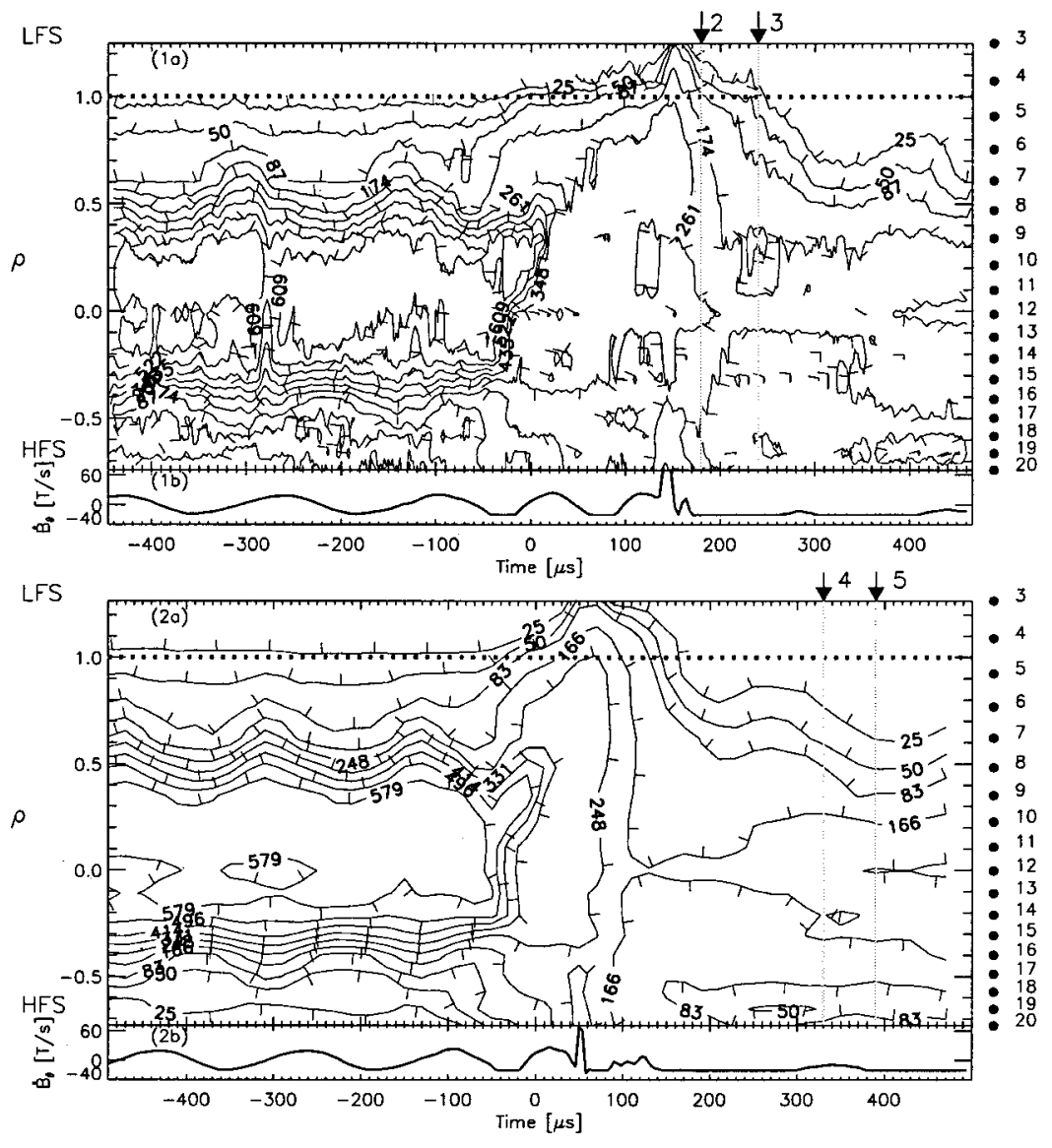

FIG. 12. The same as in Fig. 11 except that in Fig. 2(a) the time resolution is $20 \mu \mathrm{s}$. Arrows indicate the time position of TS profiles 2, 3, 4, and 5 from Fig. 5 .
${ }^{1}$ E. P. Gorbunov and K. A. Razumova, At. Energ. 15, 1105 (1963).

${ }^{2}$ F. C. Schüller, Plasma Phys. Controlled Fusion 37, A135 (1995).

${ }^{3}$ J. Wesson, R. D. Gill, and M. Hugon, Nucl. Fusion 29, 641 (1989).

${ }^{4}$ Equipe TFR, Nucl. Fusion 17, 1283 (1977).

${ }^{5}$ G. Waidman and G. Kuang, Proceedings of the 18th European Physical Society Conference on Controlled Fusion and Plasma Physics, Berlin, Germany, 1991, edited by P. Bachmann and D. C. Robinson (Europhysics Conference Abstracts, Europoean Physical Society, Petit-Lancy, 1991), Vol. 15C, p. 45.

${ }^{6}$ K. H. Finken, T. Denner, and G. Mank, Nucl. Fusion 40, 339 (2000).

${ }^{7}$ O. Gruber, K. Lackner, G. Pautasso, U. Seidel, and B. Strebl, Plasma Phys. Controlled Fusion 35, B191 (1993).

${ }^{8}$ Y. Nagayama, S. Tsuji, K. Miyamoto, K. Kawahata, N. Noda, S. Tanahashi, and J. Fujita, Proceedings of the 12th International Conference on Plasma Physics and Controlled Nuclear Fusion Research, Nice, France, 1988 [Plasma Phys. Controlled Nucl. Fusion Res. (IAEA, Vienna, Austria, 1989)], Vol. 1, p. 387.

${ }^{9}$ A. G Kellman, J. W. Cuthbertson, T. E. Evans et al., Proceedings of the 16th International Conference on Plasma Physics and Controlled Nuclear Fusion Research, Montréal, Canada, 1996 [Plasma Phys. and Controlled Nucl. Fusion Res. (IAEA, Vienna, Austria, 1997)], Vol. 1, p. 739.

${ }^{10}$ P. L. Taylor, A. G. Kellman, B. W. Rice, and D. A. Humphreys, Phys. Rev. Lett. 76, 916 (1996).

${ }^{11}$ A. C. Janos, E. D. Fredrickson, K. M. McGuire et al., Proceedings of the 14th International Conference on Plasma Physics and Controlled Nuclear Fusion Research, Würzburg, Austria, 1992 [Plasma Phys. and Controlled Nucl. Fusion Res. (IAEA, Vienna, Austria, 1993)], Vol. 1, p. 527.

${ }^{12}$ A. Bondeson, R. D. Parker, M. Hugon, and P. Smeulders, Nucl. Fusion 31, 1695 (1991).

${ }^{13}$ B. B. Kadomtsev, Plasma Phys. Controlled Fusion 26, 217 (1984)

${ }^{14}$ P. H. Diamond, R. D. Hazeltine, B. Carreras, and H. R. Hicks, Phys. Fluids 27, 1449 (1984).

${ }^{15}$ M. F. Turner and J. A. Wesson, Nucl. Fusion 22, 1069 (1982).

${ }^{16}$ B. V. Waddell, B. Carreras, H. R. Hicks, and J. A. Holmes, Phys. Fluids 22, 896 (1979).
${ }^{17}$ R. Kleva and J. Drake, Phys. Fluids B 3, 372 (1991).

${ }^{18}$ J. R. Rommers, F. C. Schüller, E. S. Lyadina, D. F. Da Cruz, M. Verreck, and A. J. H. Donné, Proceedings of the 20th European Conference on Controlled Fusion and Plasma Physics, Lisbon (European Physical Society, Petit-Lancy, 1993), Vol. 17C, Part I, 275.

${ }^{19}$ E. D. Fredrickson, K. M. McGuire, M. G. Bell et al., Nucl. Fusion 33, 141 (1993).

${ }^{20}$ F. Salzedas, Ph.D. thesis, Rijksuniversiteit Utrecht, ISBN 90-3932-571-5, 2000 (URL: http://www.library.uu.nl/digiarchief/dip/diss/1940669/ inhoud.htm).

${ }^{21}$ F. Salzedas, F. C Schüller, A. A. M. Oomens, and the RTP team, Phys. Rev. Lett. 88, 075002 (2002).

${ }^{22}$ F. C. Schüller, A. J. H. Donné, S. H. Heijnen, J. R. Rommers, C. P. Tanzi, and RTP team, in Proceedings of the 21st European Physical Society Conference on Controlled Fusion and Plasma Physics, Montpellier, France, 1994, edited by E. Joffrin, P. Platz, and P. E. Stott (Europhysics Conference Abstracts, European Physical Society, Petit-Lancy, 1994), Vol. $18 \mathrm{C}$, p. 230.

${ }^{23}$ J. D. Callen, B. V. Waddell, B. Carreras et al., Proceedings of the 7 th International Conference on Plasma Physics and Controlled Nuclear Fusion Research, Innsbruck, Austria, 1978 [Plasma Phys. Controlled Nucl. Fusion Res. (IAEA, Vienna, Austria, 1979)], Vol. 1, p. 415.

${ }^{24}$ H. P. Furth, J. Killeen, and M. N. Rosenbluth, Phys. Fluids 6, 459 (1963).

${ }^{25}$ P. H. Rutherford, Phys. Fluids 16, 1903 (1973).

${ }^{26}$ D. Biskamp, E. Schwarz, and J. F. Drake, Phys. Plasmas 4, 1002 (1997).

${ }^{27}$ J. F. Drake, D. Biskamp, and A. Zeiler, Geophys. Res. Lett. 24, 2921 (1997).

${ }^{28}$ X. H. Deng and H. Matsumoto, Nature (London) 410, 557 (1963).

${ }^{29}$ R. Kleva and P. Guzdar, Phys. Plasmas 8, 103 (2001).

${ }^{30}$ With the core facing side is meant that side of the island separatrix that is closer to the center of the plasma. Concomitantly the edge facing side is the side of the separatrix that is closer to the edge of the plasma. 